\title{
A new equation for a mass and its speed
}

\author{
Chong Wang* \\ College of Information Engineering, Zhejiang A\&F University, Lin'an, China
}

\begin{abstract}
This work showed that, when an object was accelerated, the substance quantity included in the object should remain a constant with that substance being redistributed within the object. For the same object, a different mass distribution could show a different momentum effect causing the measured mass to change. Based on this viewpoint, a new mass-speed equation was derived. Analysis showed that, without violating the law of conservation of mass, the new equation could be compatible with Special Relativity.
\end{abstract}

Keywords: Mass-speed equation; Mass distribution; Basic particle model(BPM)

\section{INTRODUCTION.}

In Special Relativity (SR), when the speed of an object changes, the motion mass of the object will vary. In addition, when the speed of the object approaches to the speed of light $c$, according to SR, the motion mass of the object will approach to infinity. Some scientists believed that a mass depends on its velocity [1][2][3]. However, other scientists considered that the mass of a body does not change with increasing velocity [4][5].

On one hand, at lower speed, the mass-speed equation of SR has been tested [6]; On the other hand, the mass conservation law has been proven repeatedly. This means that, in any situation, the substance quantity included in an object should be a constant. Then, what could cause the alteration of the mass value of an object when the speed of the object changes?

In actuality, scientists obtain the mass value of an object by detecting its momentum effect. If the substance distribution included in an object altering, the object should show a different momentum effect. It would then present a different mass value.

This research provided that, when an object is accelerated from speed 0 to $v$, the substance distribution included in the object will alter and show a stronger momentum effect. This then would cause a greater observed mass value. Based on this idea, a new mass-speed equation, being compatible with SR, was derived.

\section{NEW MASS-SPEED EQUATION.}

To obtain the relationship between a mass and its speed, a particle structure model was established.

Basic Particle Model(BPM): all basic particles (electrons, protons, neutrons etc.) consist of final particles. A final particle is the most rooted particle without structure and moves around the mass center of a basic particle at the speed of light $c$.

For the BPM, the substance included in an object belongs to the final particles. So, for an object at rest with mass $m_{0}$, the energy included in the object should be $m_{0} c^{2}$.

To obtain the mass-speed equation for an object, first the mass value, $m_{0}$, of the object at rest should be measured. Then, the object should be accelerated to speed $v$. In this process, an external force, $F$, will do some work on the object. If the object moves along a line from $r$ to $r+d r$ with the speed being changed from $v$ to $v+d v$, the work the external force done on the object, $d w$, should be:

$$
d w=F d r=\frac{d(m v)}{d t} d r=v d(m v)
$$

Where $m$ represents the substance quantity included in the object.

If the work $d w$ can cause the speed increment $d v$ for the object without changing the substance quantity included in the object, and can result in the increment of the substance quantity without changing the object speed, respectively, then, the following equation holds:

$$
d w=v d(m v)=v(m d v+v d m)
$$

Where $d m$ represents the increment of the substance quantity included in the object. In this case, Eq. 1 can be rewritten as:

$$
d w=m v d v+v^{2} d m
$$

According to the BPM, the speed of the substance included in the object keeps a constant. Thus, when the substance quantity has an increment $d m$, the energy of the object should has an increment of $c^{2} d m$. So, by the law of energy conservation, the following equation should hold: 


$$
m v d v+v^{2} d m=c^{2} d m
$$

When $v=0, m=m_{0}$ holds. So, the solution of Eq. 3 can be obtained:

$$
m=\frac{m_{0}}{\sqrt{1-\left(\frac{v}{c}\right)^{2}}}
$$

Eq. 4 is equal to the mass-speed equation of SR. But it conflicts with the law of mass conservation.

Since the law of conservation of mass has been repeatedly verified, the substance quantity included in an object should be constant when the speed of the object varies. This means that, for the right side of Eq. 2 , the increment of the substance quantity included in the object, $d m$, should be zero.

However, when the object has a speed increment of $d v$, a simultaneous mass redistribution within the object will be produced. This will cause a stronger momentum effect, meaning that a speed increment $d v$ will bring about an observed mass value increment $d m^{*}$ without additional work.

Nevertheless, $d m^{*}$ does not belong to a real substance included in the object. Rather it is just an appearance of the stronger momentum effect. This $d m^{*}$ is called a motion mass increment, and it results only from the substance redistribution.

Therefore, it can be said that when an object is accelerated from 0 to $v$, its mass will increase from $m_{0}$ to $m^{*}$. In this case, $m^{*}$ is called the motion mass.

For an object moving along a straight line, the momentum increment of the object must cost a work on it. When we accelerate the object from speed $v$ to $v+d v$, its motion mass $m^{*}$ should become $m^{*}+d m^{*}$ automatically without consuming additional work.

According to above analysis, the momentum increment of the object, $d\left(m^{*} v\right)$, should be

$$
d\left(m^{*} v\right)=\left(m^{*}+d m^{*}\right) d v
$$

So, the work, $d w$, done by the external force $F$ should be

$$
d w=F d r=\frac{d\left(m^{*} v\right)}{d t} d r=v\left(m^{*}+d m^{*}\right) d v
$$

By the BPM, the energy increment should be $c^{2} d m^{*}$. Thus, by the law of energy conservation and by omitting $v d m^{*} d v$, the following equation is produced:

$$
\frac{d m^{*}}{m^{*}}=\frac{v}{c^{2}} d v
$$

Considering that, when $v=0, m^{*}=m_{0}$ holds, the solution of eq. 7 can be obtained:

$$
m^{*}=m_{0} e^{\frac{v^{2}}{2 c^{2}}}
$$

The first order approximation for Eq. 8 is:

$$
m^{*}=m_{0}\left(1+\frac{v^{2}}{2 c^{2}}\right) \approx \frac{m_{0}}{\sqrt{1-\left(\frac{v}{c}\right)^{2}}}
$$

When $v$ has low value, Eq. 8 and Eq. 9 are equal.

Therefore, it can be said that, at low speed, Eq. 8 is compatible with the mass-speed equation of SR.

However, by Eq. 8, when $v \rightarrow c$, different from SR, the motion mass of an object is a constant:

$$
m^{*}=m_{0} \sqrt{e}=1.65 m_{0}
$$

Experiments showed that [7], for electrons, when $v \rightarrow c$, $m^{*} / m_{0}$ approaches to a constant 1.45. Thus, we can said that, when $v \rightarrow c$, Eq. 8 is more compatible with the experiment than SR.

\section{CHECKING THE BASIC PARTICLE MODEL(BPM) USING SPECIAL RELATIVITY.}

Based on the basic particle model (BPM), a new mass-speed equation Eq. 8, compatible with SR, has been derived. If the BPM is correct, it should be compatible with the other results of SR. For example, it should be compatible with the Mass-Energy Equation, the Length-Contraction Effect, and the Time-Dilation Effect etc.

(a) Mass-Energy Equation: By the BPM, the energy $E$, of a motion mass $m^{*}$, should be:

$$
E=m^{*} c^{2}
$$

So, it can be said that, the BPM is completely compatible with the mass-energy equation of SR.

\section{(b) Length Contraction Effect:}

For a ruler at rest, its length $l_{0}$ corresponds to its inner energy $m_{0} c^{2}$. If it is accelerated from speed $v$ to $v+d v$, the work $d w$ done on it will shrink the ruler by a 
value of $d x$. This means that, $-d x$ corresponds to $d w$. Thus, the following equation is obtained:

$$
\frac{l_{0}}{m_{0} c^{2}}=-\frac{d x}{d w}
$$

Using Eq. 6 and omitting the higher order item produces:

$$
d x=-\frac{l_{0}}{m_{0} c^{2}} m^{*} v d v
$$

Using Eq. (8), the solution of Eq. 12 is obtained:

$$
x=l_{0}\left(2-e^{\frac{v^{2}}{2 c^{2}}}\right)
$$

The first order item of Eq. 13 is:

$$
x=l_{0}\left(1-\frac{v^{2}}{2 c^{2}}\right) \approx l_{0} \sqrt{1-\left(\frac{v}{c}\right)^{2}}
$$

So, by Eq. 13, it can be concluded that, at low speed, the BPM is compatible with the length contraction effect of SR.

(c) Time Dilation Effect: In our daily life, time is measured only by cyclic motion. For example, the time the earth takes to complete a revolution around the sun is called a year. The time the moon takes to complete a revolution around the earth is called a month. So, the uniform circular motion of a mass $m$, can be viewed as a clock system. Where $m$ is called the clock mass.

If a clock system is in an inertial system and stays in position $P$ (called a clock at rest), then it can be used to measure the time of an object moving in the inertial system. For a clock system at rest, in an average, the clock mass should have no substance redistribution when it completes a cycle. So, its value can be viewed as $m_{0}$. Where $m_{0}$ is the mass value when the clock mass stops its cyclic motion. If the clock system moves along a straight line from position $P$ to another position $P^{\prime}$, an external force $F$ must act on the clock system. Then, the clock mass will have a substance redistribution.

Suppose the work done by $F$ on the clock system is $d w$. Then, the clock speed will change from $v$ to $v+d v$ as the clock moving from $P$ to $P^{\prime}$. During this process, the mass of the clock will have a longer motion path (from a circle to an oval), and thus produces a time increase $d t$.

For the clock system at rest, if $m_{0} c^{2}$ corresponds to a time span of $t_{0}$, then, $d w$ should correspond to a time span of $d t$, meaning that the following equation holds:

$$
\frac{t_{0}}{m_{0} c^{2}}=\frac{d t}{d w}
$$

Combining Eq. 6, Eq. 8, and Eq. (15), and omitting the higher order item produces:

$$
d t=t_{0} e^{\frac{v^{2}}{2 c^{2}}} d \frac{v^{2}}{2 c^{2}}
$$

The solution of Eq. 16 is:

$$
t=t_{0} e^{\frac{v^{2}}{2 c^{2}}}
$$

Eq. 17 shows that, for a moving clock system, the time the clock mass takes to complete a cyclic motion is longer than the clock system at rest. Meaning that the number displayed by the moving clock is less than the clock at rest.

The first order approximation for Eq. 17 is

$$
t=t_{0}\left(1+\frac{v^{2}}{2 c^{2}}\right) \approx \frac{t_{0}}{\sqrt{1-\left(\frac{v}{c}\right)^{2}}}
$$

Eq. 18 indicates that, at low speed, the BPM is compatible with the time-dilation effect of SR.

\section{CONCLUSION.}

When the speed of an object changes, the substance distribution included in the object will be simultaneously altered. This causes the motion mass of the object. Different from Special Relativity, when the speed of the object approaches the speed of light, the motion mass of the object becomes a constant. And the relationship between the mass of the object and its speed obey Eq. 8.

* 390566584@qq.com

[1] Born M., Einstein's Theory of Relativity (New York: DoverPubl., 1962) p. 269.

[2] Feynman R., Character of Physical Law (London: Cox and Wyman, 1965) p. 76.

[3] Strelkov S. P. , Mechanics (Moscow: Nauka, 1975) p. 533 (in Russian).

[4] Okun' L. B. , Physics Today 42 (6), 31 (1989).

[5] Taylor E. F., Wheeler J. A., Spacetime Physics (San Francisco: W. H. Freeman, 1966).

[6] W. Bertozzi, Am. J. Phys. 32. 551 (1964).

[7] Ji Hao, Chinese scientific and technoligical achievements, In Chinese, 2009(1). 